\title{
Clear Cell Carcinoma of the Uterine Cervix Arising from an Interstitial Cyst Complicated with Endometriosis: A Case Report
}

\section{Kana Akagi, Takako Tobiume, Ayaka Kawamichi, Yuichiro Koshida, Yusuke Fujikami, Eri Ogura, Kenji Ban, Hisanori Matsumoto, Atsuhiko Okagaki, Keiji Tatsumi*}

Department of Obstetrics and Gynecology, National Hospital Organization Osaka National Hospital, Osaka, Japan

Email: *tatsumi.keiji.qb@mail.hosp.go.jp

How to cite this paper: Akagi, K., Tobiume, T., Kawamichi, A., Koshida, Y., Fujikami, Y., Ogura, E., Ban, K., Matsumoto, H., Okagaki, A. and Tatsumi, K. (2021) Clear Cell Carcinoma of the Uterine Cervix Arising from an Interstitial Cyst Complicated with Endometriosis: A Case Report. Open Journal of Obstetrics and Gynecology, 11, 288-295.

https://doi.org/10.4236/ojog.2021.113028

Received: February 10, 2021

Accepted: March 15, 2021

Published: March 18, 2021

Copyright $\odot 2021$ by author(s) and Scientific Research Publishing Inc. This work is licensed under the Creative Commons Attribution International License (CC BY 4.0).

http://creativecommons.org/licenses/by/4.0/

\section{(c) (i) Open Access}

\begin{abstract}
Clear cell carcinoma (CCC) of the uterine cervix is rare, and its etiology is unclear, except for reports of prenatal diethylstilbestrol (DES) exposure. In this case report of a 78-year-old postmenopausal Japanese woman with pelvic endometriosis, cervical CCC presented as a deep interstitial tumor, strongly suggesting the patient suffered from cervical endometriosis for a long time. A cystic lesion without solid components was detected in the uterine cervix. Although the patient had regular gynecological examinations every 6 months, she presented 5 years later with a complaint of watery vaginal discharge. Ultrasonography and magnetic resonance imaging (MRI) detected a uterine cervical cystic mass with a solid component. Although no malignancy was confirmed preoperatively, surgery was performed under a high suspicion of cervical cancer. Intraoperative pathology revealed CCC in a solid tumor, so debulking surgery, including lymphadenectomy, was done. Endometrial tissue was found adjacent to the tumor, strongly suggesting the tumor arose from cervical endometriosis. Because endometriosis in ectopic sites carries a risk of carcinogenesis, as is the case in ovarian endometriosis, it may be recommended that a cervical cystic lesion with pelvic endometriosis should be followed up regularly for the early detection of uterine cancer.
\end{abstract}

\section{Keywords}

Cancer, Clear Cell Carcinoma, Endometriosis, Uterine Cervix

\section{Introduction}

Clear cell carcinoma (CCC) is an adenocarcinoma composed of glycogen-rich 
clear cytoplasm cells, or cells that have a hobnail-like morphology with small cytoplasm and large nuclei. Adenocarcinoma accounts for $20 \%-25 \%$ of uterine cervical cancer, and CCC is so rare that it accounts for only $4 \%-9 \%$ of cervical adenocarcinoma [1]. It has been reported that intrauterine DES exposure could have induced outbreaks of vaginal and cervical CCC [2] [3]. One report showed CCC patients without exposure to DES were relatively older; however, another report demonstrated twin peaks in the ages of non-DES-exposed CCC patients [3]. The factors involved in the initiation and progression of CCC are still unknown, although cervical endometriosis has been suggested [4] [5]. Here we report a rare case of CCC arising from an intramural cyst of the uterine cervix complicated with endometriosis despite 5 years of regular gynecological visits.

\section{Case Presentation}

A 78-year-old postmenopausal nulliparous Japanese woman presented to our hospital in the year X with a complaint of genital bleeding. She had been diagnosed with endometriosis by a gynecologic clinic at the age of 24 , and the contents of the ovarian endometrial cysts were extracted by transvaginal puncture in the following year. She had no history of prenatal DES exposure and did not receive any hormone replacement therapy; however, she had drug-induced fulminant hepatitis at 71 years. Her family history was unremarkable. The patient's onset of menarche occurred at 13 years of age and menopause at 47 years. She had suffered from dysmenorrhea and hypermenorrhea throughout her reproductive years. On gynecological examination, no significant lesion was found in the external genitalia or vagina, and the uterine cervix was clean without genital bleeding. Transvaginal ultrasonography showed an endometrial thickness of 9 $\mathrm{mm}$ and a cystic lesion measuring $7 \times 13 \mathrm{~mm}$ in the cervical wall (Figure 1(a)). Magnetic resonance imaging (MRI) showed a cystic lesion of high intensity on the T2-weighted image without a solid component (Figure 1(b)). The uterus was strongly anteflexed with multiple small myomas. There was a $5 \mathrm{~mm}$ nodular mass in the endometrial cavity, suggesting an endometrial polyp. The ovaries appeared normal bilaterally. The rectum was adhered to the posterior wall of the uterus, and Douglas' pouch was closed. A hysteroscopy was done, and pathology of the endometrial mass did not detect any atypical cells. Afterward, the patient came to our hospital for regular gynecological examinations every 6 months for 5 years. During this time, she remained asymptomatic, and the cervical cystic lesion was unchanged.

In the year $X+5$, she presented to us with a complaint of watery vaginal discharge. Upon examination, the surface of the uterine cervix was smooth, but the posterior lip was bulging, and serous fluid was flowing out of a small hole at 7 o'clock. Transvaginal ultrasonography showed a $50 \times 51 \times 49 \mathrm{~mm}$ cystic lesion in the uterine cervix with a solid component (Figure 2(a)). Enhanced MRI demonstrated the cervical mass consisted of irregular solid and cystic components, and the solid components showed hyperintensity (Figure 2(b)) as well as 


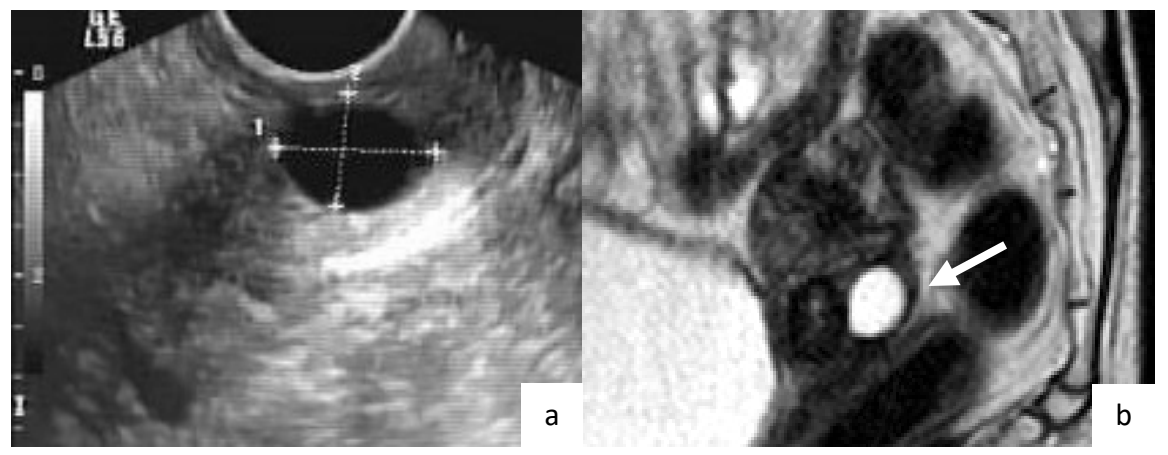

Figure 1. Images of the uterine cervical cyst at the initial visit. (a) $7 \times 3 \mathrm{~mm}$ cystic lesion in the cervical wall. (b) Pelvic MRI showed a cystic lesion with high intensity on the T2-weighted image without a solid component.

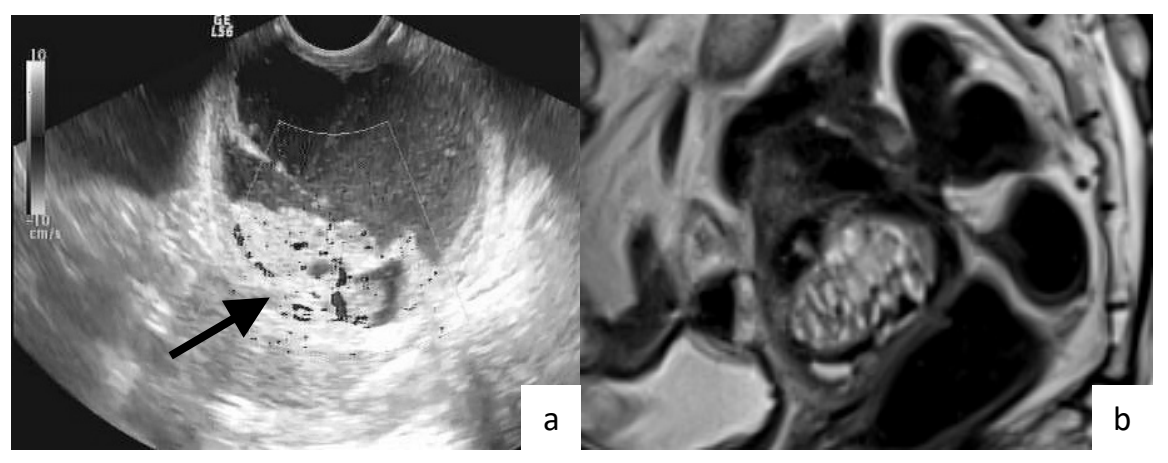

Figure 2. Images of the uterine cervical cyst in the year $X+5$. (a) Transvaginal ultrasonog raphy showed a cystic lesion of $50 \times 51 \times 49 \mathrm{~mm}$ in the uterine cervix containing a solid component (arrow). (b) Enhanced MRI demonstrated the cervical mass consisted of solid and cystic components. The solid components had irregular margins and exhibited strong enhancement.

high signal on a diffusion-weighted MRI. The tumor margin was clear, and no invasion beyond the uterine cervix was observed. A Pap smear of the fluid in the cyst detected only lymphocytes. A pathological examination of the tumor through aspiration showed stratified squamous epithelial tissue, including a keratinizing part. There was neither nuclear deformity nor any malignant finding. All examined tumor markers, including carcinoembryonic antigen (CEA), carbohydrate antigen 19-9 (CA19-9), cancer antigen 125 (CA125), $\alpha$-fetoprotein (AFP), and squamous cell carcinoma antigen (SCC), were within the normal ranges.

A hysteroscopy showed no abnormal findings in the cervix, and intracervical curettage detected no malignant tissue. Under the diagnosis of a uterine tumor suspicious for cervical cancer, a total hysterectomy with bilateral salpingo-oophorectomy was performed. Because the intraoperative pathology showed adenocarcinoma, para-aortic and pelvic lymphadenectomy and subtotal omentectomy were added. A rapid intraoperative cytology test was negative, and no disseminated lesions were found in the abdominal cavity. There was strong adhesion between the uterus and rectum, suggesting pelvic endometriosis. Al- 
though the surface of the cervical canal in the specimen was smooth, a solid and encapsulated component was revealed in the posterior wall (Figure 3). The cystic tumor had ruptured only at the vaginal site. Histologically, the tumor cells were characterized by clear cytoplasm and a hobnail-like appearance, which led to the diagnosis of CCC (Figure 4(a) and Figure 4(b)). The tumor occupied almost the entire posterior wall of the cervix, but its border was clear, and no invasion of the wall was observed. There was neither lymphatic nor venous infiltration, and metastases were not detected in the lymph nodes or the omentum. Adenomyotic tissue was found in the uterine corpus (Figure 4(c)). Endometrial epithelial and stromal cells were also detected on the exfoliated surface of the posterior wall of the cervix (Figure 4(d)) and near the tumor (Figure 4(e) and Figure 4(f)), confirming the presence of endometriosis. The patient was diagnosed with cervical CCC stage 1B1 (pT1b1N0M0), according to the 2008 International Federation of Gynecology and Obstetrics (FIGO) staging. Postoperative

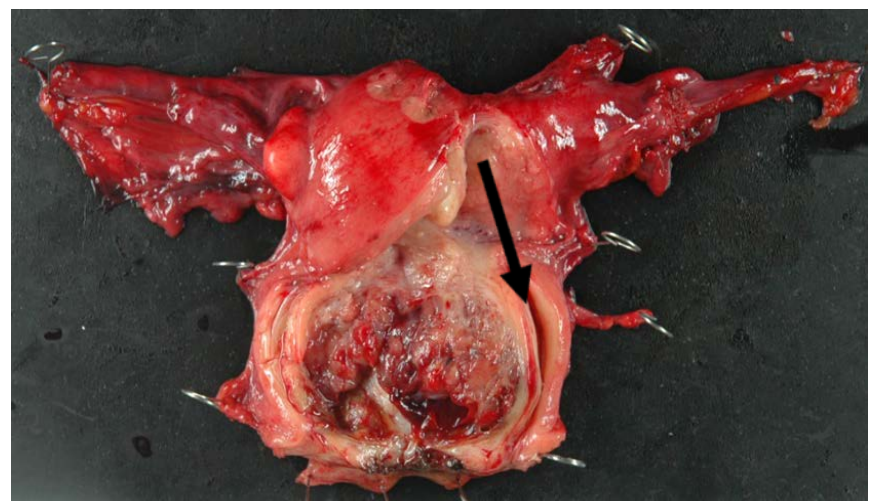

Figure 3. Surgical specimen of the uterus. A solid and encapsulated compon ent $(5 \times 5 \times$ $5 \mathrm{~cm}$ ) was revealed in the posterior wall. The surface of the cervical canal was smooth (arrow).

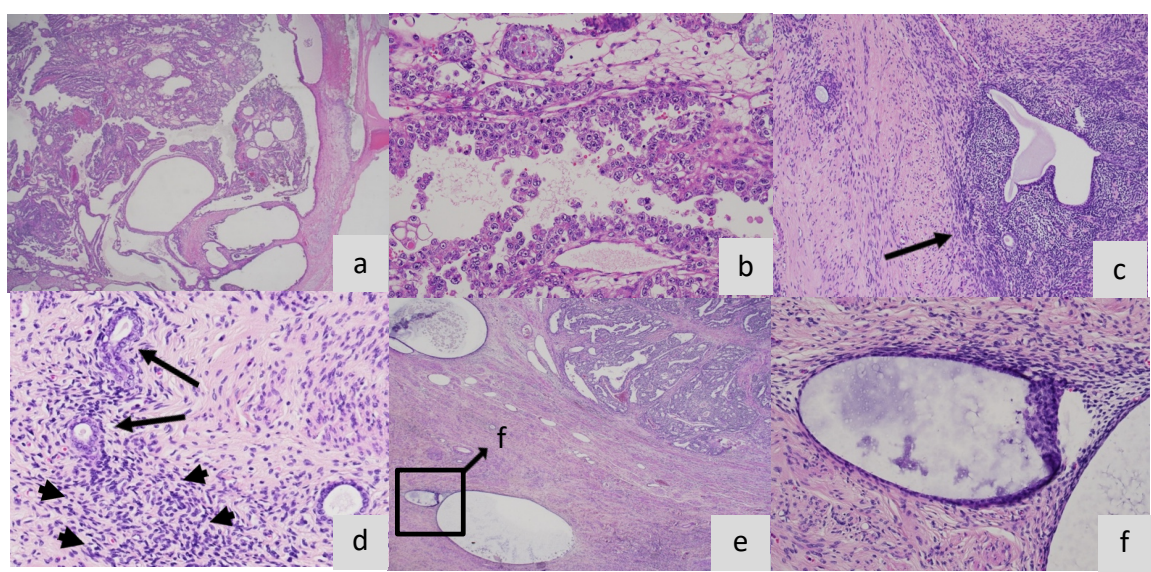

Figure 4. Histology of the tumor. Tumor cells have clear cytoplasm and a hobnail-like appearance that is distinctive in CCC $((\mathrm{a}) \times 40,(\mathrm{~b}) \times 400)$. Adenomyotic tissue (arrow) was found in the uterine corpus $((c) \times 100)$. Endometrial epithelial cells (arrows) and stromal cells (arrowheads) were also detected at the exfoliated surface of the posterior wall $((\mathrm{d}) \times 200)$ and near the tumor $((\mathrm{e}) \times 40$, (f) $\times 200)$ 
course had been uneventful except for a mild inflammation, which improved promptly by administration of antibiotics. After the surgery, chest and abdominal computed tomography showed no residual lesions or metastasis. Because the patient was at high risk for recurrence, she was subsequently treated with carboplatin and paclitaxel for adjuvant chemotherapy. After the 6 cycles of chemotherapy, the general condition of the patient has been good and no recurrent symptom has been observed.

\section{Discussion}

In Japan, CCC is rare and accounts for only $4 \%$ of adenocarcinoma of the cervix [1]. It has been reported that intrauterine exposure to DES for preventing miscarriage could have induced CCC. However, DES was not clinically used in Japan, and only 18 cases have been reported so far ( 2 cases in young patients and 16 cases in elderly patients). Although one report showed CCC patients without exposure to DES were relatively older, another report demonstrated twin peaks in the ages of non-DES-exposed CCC patients [3]. The factors involved in the initiation and progression of CCC are still unknown, and a treatment strategy has not been established because of the small number of CCC cases.

CCC cases exposed to DES tend to show exophytic growth, while those cases without exposure to DES tend to have endophytic growth [6]. Reich et al. analyzed 15 CCC cases exposed to DES and compared them with 444 SCC and 59 non-clear cell adenocarcinoma (NCCA) cases. The CCC cases showed predominantly endophytic growth and extended to the uterine corpus significantly more often than did the SCC and NCCA cases [7]. There were 2 cases of CCC that occurred in the cervical interstitial tissue without infiltration to the intracervical epithelium [4] [5]. In each case, a background of cervical endometriosis was detected. Uterine cervical adenocarcinoma usually occurs in the glandular epithelium and progresses deep into the wall, while cervical CCC arising from endometriosis may occur in deep interstitial regions and progresses into the cervical canal.

The chief complaint in most cases of cervical adenocarcinoma is genital bleeding, which is similar to that of CCC [8]. It has been suggested that in many CCC cases, the tumor progresses to form punctate ulcers and low ridges in the cervical lumen. However, cases of interstitial CCC without invasion of the lumen often lack genital bleeding or other symptoms. In the present case, the patient had received gynecological examinations every 6 months for 5 years, and the cystic lesion of the cervix had not changed until the patient became aware of watery discharge 5 months after her most recent examination. However, she waited to see us for about another month because the fluid was not bloody and she had already scheduled a regular 6-month follow-up appointment. Without regular follow-up, her consultation might have been delayed, and the tumor could have progressed further.

Another factor that makes the diagnosis of CCC difficult is the low positive 
rate of cytology. Thomas reported that abnormal cytology was noted in $6(18 \%)$ of 31 patients with CCC [7]. It has been suggested that this low positive rate of cytology is because of the endophytic growth of the tumor and the lack of cervical luminal invasion. In the present case, cervical cytology remained normal every 6 months during the 5 years of follow-up, probably because the tumor had not invaded the cervical lumen. However, despite the rupture of the cyst containing the tumor, neither the cytology of the fluid in the cyst nor the histology of the tumor through aspiration revealed malignant cells. Thus, no definitive diagnosis of malignancy was made before surgery. It is not clear why the biopsy did not reveal malignancy, but it is supposed that the CCC tumor was encapsulated in the specimen, and the tumor cells had not spread in the cyst. This possibility is supported by the fact that the fluid was not bloody despite the tumor arising from cervical endometriosis. When a tumor is highly suspicious for malignancy, great care should be taken in performing the biopsy to obtain a specimen precisely.

Ovarian endometriosis is a factor that puts patients at high risk for ovarian cancer, especially CCC and endometrioid carcinoma [9]. Previous reports have described an association between cervical endometriosis and CCC [4] [5], and there are some case reports of CCC arising from ectopic endometriosis in the vagina, abdominal wall, or rectum [10] [11] [12] [13], although they are less frequent than those in the cervix. The mechanism of carcinogenesis of ovarian endometrial cysts is not clear, but some theories have been advocated, such as DNA damage caused by oxidative stress due to the iron component [14], gene mutations of ARIDIA or PIK3CA, and exposure to environmental factors such as high estrogen, inflammation, and tumor immunity [15]. The theory of exposure to a high-estrogen environment because of endometriosis is common to carcinogenesis caused by exposure to DES; thus, this theory may also explain the carcinogenesis of ectopic and ovarian endometriosis. Sterns et al. reported that there were many more synchronous uterine tumors in the cases of ovarian cancer derived from ectopic endometriosis than in the cases without endometriosis, suggesting that the ectopic endometrium may also undergo similar estrogendependent changes as the endometrium [9]. In our case, it was difficult to diagnose cervical endometriosis in the cystic lesion. The solid mass was shown on MRI as a low-intensity area in the T1-weighted image and a high-intensity area in the T2-weighted image. Endometriotic tissue found on the exfoliated surface of the posterior wall of the cervix revealed the presence of at least pelvic endometriosis. Histologically, endometrial tissue was detected in the posterior wall of the uterine cervix very close to the tumor, and adenomyotic tissue was found in the uterine corpus. The growth center of the tumor deep in the cervical interstitium strongly suggests the tumor developed from cervical endometriosis. Further analysis of the carcinogenic mechanism of ovarian endometriotic cysts may reveal a link between CCC and cervical endometriosis.

The standard therapy for CCC has not been established and is currently based 
on the methods for SCC and NCCA. In the early stages, radical hysterectomy is recommended. Radical trachelectomy has emerged as a viable option in the early stages (IA-IB1) of CCC without lymphatic spread for women younger than 45 years who have a strong desire to preserve fertility [16]. Reich et al. reported that CCC had a lower 5-year survival rate (67\%) than SCC (80\%) and NCCA (77\%), although it was not statistically significant $(\mathrm{p}=0.6)$ [7]. It has been reported that stage I or IIA patients had a better 3-year overall survival rate compared to stage III or IV patients ( $92 \%$ vs. $22 \%, \mathrm{p}<0.001$ ), and the FIGO stage was an important prognostic factor. Thus, early detection of tumors is very important to improve the prognosis. In the present case, regular follow-up for the patient's cervical cystic lesion led to the early detection of CCC. Future studies may suggest new, cost-effective management strategies for cervical cystic lesions with pelvic endometriosis.

\section{Conclusion}

We report a case of CCC arising from a cervical cystic lesion with pelvic endometriosis. This case suggests cervical endometriosis was involved in the development of CCC. Ectopic endometriosis, like ovarian endometrial cysts, may carry a risk of future carcinogenesis, and regular follow-up for cervical cystic lesions in patients with endometriosis would be recommended.

\section{Consent}

Informed consent was obtained.

\section{Conflicts of Interest}

The authors have no conflicts of interest relevant to this article.

\section{References}

[1] Hasegawa, K., Nagao, S., Yasuda, M., Millan, D., Viswanathan, A.N., Glasspool, R.M., Devouassoux-Shisheboran, M., Covens, A., Lorusso, D., Kurzeder, C., Kim, J.W., Gladieff, L., Bryce, J., Friedlander, M. and Fujiwara, K. (2014) Gynecologic Cancer InterGroup (GCIG) Consensus Review for Clear Cell Carcinoma of the Uterine Corpus and Cervix. International Journal of Gynecological Cancer, 24, S90-S95. https://doi.org/10.1097/IGC.0000000000000297

[2] Herbst, A.L., Ulfelder, H. and Poskanzer, D.C. (1971) Adenocarcinoma of the Vagina: Association of Maternal Stillbestrol Therapy with Tumor Appearance in Young Women. New England Journal of Medicine, 284, 878-881. https://doi.org/10.1056/NEJM197104222841604

[3] Hanselaar, A., van Loosbroek, M., Schuurbiers, O., Helmerhorst, T., Bulten, J. and Bernhelm, J. (1997) Clear Cell Adenocarcinoma of the Vagina and Cervix. An Update of the Central Netherlands Registry Showing Twin Age Incidence Peaks. Cancer, 79, 2229-2236. https://doi.org/10.1002/(SICI)1097-0142(19970601)79:11<2229::AID-CNCR22>3.0. CO;2-X

[4] Hashiguchi, M., Kai, K., Nishiyama, S., Nakao, Y., Yokoyama, M. and Aishima, S. 
(2018) Clear Cell Carcinoma of the Uterine Cervix Presented as a Submucosal Tumor Arising in a Background of Cervical Endometriosis. International Journal of Gynecological Pathology, 37, 88-92.

https://doi.org/10.1097/PGP.0000000000000386

[5] Hiromura, T., Tanaka, Y.O., Nishioka, T., Satoh, M. and Tomita, K. (2009) Clear Cell Adenocarcinoma of the Uterine Cervix Arising from a Background of Cervical Endometriosis. British Journal of Radiology, 82, e20-e22. https://doi.org/10.1259/bjr/75304693

[6] Fukumura, N. (2017) The General Rules for Clinical and Pathological Management of Uterine Cervical Cancer. Vol. 4, Pathological Edition, Kanehara Publishing, Tokyo, 39.

[7] Reich, O., Tamussino, K., Lahousen, M., Pickel, H., Haas, J. and Winter, R. (2000) Clear Cell Carcinoma of the Uterine Cervix: Pathology and Prognosis in Surgically Treated Stage IB-IIB Disease in Women Not Exposed in Utero to Diethylstilbestrol. Gynecologic Oncology, 76, 331-335. https://doi.org/10.1006/gyno.1999.5700

[8] Sandberg, E.C., Danielson, R.W., Cauwet, R.W. and Bonar, B.E. (1965) Adenosis Vaginae. American Journal of Obstetrics and Gynecology, 93, 209-222.

https://doi.org/10.1016/0002-9378(65)90660-5

[9] Stern, R.C., Dash, R., Bentley, R.C., Snyder, M.J., Haney, A.F. and Robboy, S.J. (2001) Malignancy in Endometriosis: Frequency and Comparison of Ovarian and Extraovarian Types. International Journal of Gynecological Pathology, 20, 133-139. https://doi.org/10.1097/00004347-200104000-00004

[10] Achach, T., Rammeh, S., Trabelsi, A., Ltaief, R., Abdelkrim, S.B., Mokni, M. and Korbi, S. (2008) Clear Cell Adenocarcinoma Arising from Abdominal Wall Endometriosis. Journal of Oncology, 2008, Article ID: 478325. https://doi.org/10.1155/2008/478325

[11] Bolis, G.B. and Maccio, T. (2000) Clear Cell Adenocarcinoma of the Vulva Arising in Endometriosis. A Case Report. European Journal of Gynecologic Oncology, 21, 416-417.

[12] Shah, C., Pizer, E., Veljovich, D.S., Drescher, C.W., Peters, W.A. and Paley, P.J. (2006) Clear Cell Adenocarcinoma of the Vagina in a Patient with Vaginal Endometriosis. Gynecologic Oncology, 103, 1130-1132. https://doi.org/10.1016/j.ygyno.2006.06.044

[13] Okazawa, Y., Takahashi, R., Mizukoshi, K., Takahara, K., Ishiyama, S., Sugimoto, K., Takahashi, M., Kojima, Y., Goto, M., Okuzawa, A., Tomiki, Y., Yao T. and Sakamoto, K. (2014) A Case of Clear Cell Adenocarcinoma Arising from Endometriosis of the Rectum Treated by Laparoscopic Surgery. International Journal of Surgical Case Reports, 5, 979-983. https://doi.org/10.1016/j.ijscr.2014.10.034

[14] Vercellini, P., Viganò, P., Somigliana, E. and Fedele, L. (2014) Endometriosis: Pathogenesis and Treatment. Nature Reviews Endocrinology, 10, 261-275. https://doi.org/10.1038/nrendo.2013.255

[15] Oda, K., Hamanishi, J., Masuo, K. and Hasegawa, K. (2018) Genomics to Immunotherapy of Ovarian Clear Cell Carcinoma: Unique Opportunities for Management. Gynecologic Oncology, 151, 381-389. https://doi.org/10.1016/j.ygyno.2018.09.001

[16] Gien, L.T. and Covens, A. (2010) Fertility-Sparing Options for Early Stage Cervical Cancer. Gynecologic Oncology, 117, 350-357. https://doi.org/10.1016/j.ygyno.2010.01.039 